\title{
RUSSELL Y HEGEL, UNA DISCUSIÓN SOBRE DEÍCTICOS
}

\author{
Susana Gómez Gutiérrez* \\ doi:10.11144/Javeriana.uph33-66.rhdd
}

\begin{abstract}
RESUMEN
En este trabajo examino las maneras contrapuestas como Bertrand Russell, en varios de sus escritos, y Hegel, en la Fenomenología del espiritu, entienden los llamados deícticos. Intento mostrar que Russell, aun conociendo bien la obra de Hegel, no responde a la propuesta de este último, sino que adopta una posición que lo mantiene atrapado en lo que, desde un punto de vista hegeliano, podría denominarse el autoengaño de la certeza sensible. Al final, sugiero que las diferencias entre Hegel y Russell, a propósito de los deícticos, pueden deberse al hecho de que ambos adoptan ontologías que se excluyen la una a la otra.

Palabras clave: deícticos; Hegel; Russell; filosofía de la substancia; filosofía de procesos
\end{abstract}

Corporación Universitaria Minuto de Dios, Bogotá, Colombia.

Correo electrónico: batata.susana@gmail.com

Para citar este artículo: Gómez GuTiéRRez, S. (2016). Russell y Hegel, una discusión sobre deícticos. Universitas Philosophica, 33(66), pp. 113-127. ISSN 0120-5323, ISSN en línea: 2346-2426, doi:10.11144/Javeriana.uph33-66.rhdd

Agradezco al profesor Roberto Solarte, a mis compañeros del seminario de doctorado Hegel, La fenomenologia del espiritu de la Pontificia Universidad Javeriana, a los profesores Juan Carlos Guerrero, Jorge Aurelio Díaz, Miguel Ángel Pérez, a los dos pares evaluadores anónimos sus comentarios a este escrito y la discusión de algunos temas tratados aquí. Agradezco muy especialmente a mis colegas John Larry Rojas, quien se leyó todas las versiones previas de este texto, y con paciencia lo corrigió y me hizo darle la vuelta una y otra vez, y a José Andrés Forero, cuyos comentarios me obligaron a darle la vuelta final. Sobra decir que, pese a todas las ayudas anteriores, cada error que contenga este texto es únicamente de mi responsabilidad. Y, finalmente, como siempre, agradezco a Dios, a la Virgen, a los ángeles y a los santos por todo. 


\title{
RUSSELL AND HEGEL, A DISCUSSION ON DEICTICS
}

\author{
Susana Gómez Gutiérrez
}

\begin{abstract}
In this paper I examine the conflicting ways in which Bertrand Russell and Hegel understand indexical expressions. For this purpose, I consider some books and articles written by Russell in different moments of his life, and the first chapter of Hegel's Phenomenology of Spirit, book published in 1807. I try to show that Russell, even being a Hegel scholar, does not respond to his proposal about indexical expressions, but takes a position that keeps him trapped in a kind of self-deception of sense-certainty. At the end, I suggest that the differences between Hegel and Russell in relation to indexical expressions can be explained by the fact that the ontologies adopted by both authors exclude each other.

Key words: indexical expressions; Hegel; Russell; substance philosophy; process philosophy
\end{abstract}


En la Historia INTELECTUAL de Bertrand Russell, bien conocido es el hecho de su adhesión inicial al idealismo de Hegel y al de los llamados Idealistas Británicos, entre los que se cuentan a F.H. Bradley y J.M.E. McTaggart. Bien conocido también es su posterior rechazo de tales doctrinas, siendo su Filosofía del Atomismo Lógico (FAL), publicada en 1918, una especie de manifiesto anti-idealista. En resumen, podemos decir que Russell, en tanto realista, rechaza la idea hegeliana de que la realidad es una sola, ya que a esta contrapone la doctrina según la cual el mundo "se concibe como compuesto por muchas entidades independientes distintas, cada una de las cuales puede ser considerada aisladamente de sus relaciones con otras cosas y de sus relaciones con la mente" (Klement, 2014, p. 8). Tales entidades las constituyen los hechos atómicos, que hipotéticamente serían el correlato metafísico de lo que en el lenguaje corresponde a las proposiciones atómicas ${ }^{1}$. Sin embargo, en otros pasajes parece referir a entidades aún más elementales, aquellas que constituyen el significado de lo que, en el lenguaje, el autor llama 'símbolos simples'.

En Misticismo y Lógica (ML), obra de 1917, Russell habla del conocimiento por familiaridad como aquel que no está mediado por ningún tipo de inferencia o de concepto, y del conocimiento por descripción como aquel en el que conocemos a un individuo x solo por medio de una descripción de la forma 'el tal y tal', esto es, una descripción que solo a él corresponde (Russell, 1973, pp. 10301033). No obstante, para Russell, esa descripción está, a su vez, formada por términos con los cuales tenemos conocimiento por familiaridad, pues "toda proposición que podemos comprender ha de componerse enteramente de constitutivos con los que estamos familiarizados" (Russell, 1973, p. 1035). En este punto, los constitutivos se entienden como los referentes de los símbolos simples, esto es, los referentes de los nombres propios. Con esto, surge la pregunta: ¿qué son esos referentes de los nombres propios, aquello de lo cual tenemos

1 Las proposiciones atómicas se unen entre sí a través de los conectores lógicos, formando las proposiciones moleculares. Ejemplos de proposiciones atómicas serían: 'el gato está sobre la alfombra,' 'el gato juega con un ratón', en las cuales no aparecen conectores lógicos. Ejemplos de proposiciones moleculares serían: 'el gato está sobre la alfombra y juega con un ratón', 'el gato está sobre la alfombra o juega con un ratón', etc., en las cuales sí aparecen dichos conectores. 
conocimiento por familiaridad? Una idea muy común en Russell es que aquello a lo que nosotros gramaticalmente llamamos nombres propios como 'Sócrates' y 'Platón' no son en realidad nombres propios, pues no tenemos conocimiento por familiaridad de sus objetos, sino que son descripciones definidas disfrazadas que no describen individuos particulares, sino "complicados sistemas de clases o de series" (Russell, 1981, p. 281). Para él, lo único de lo que tenemos conocimiento por familiaridad es aquello a lo que nos referimos cuando decimos 'esto es blanco' o 'aquello es blanco' y, por lo tanto, las partículas lingüísticas que funcionarían como nombres propios en el sentido lógico del término (nombres estrictos) serían 'esto' o 'aquello, y sus referentes, es decir, las entidades correspondientes serían las entidades particulares a las cuales apunten 'esto' o 'aquello' en una circunstancia específica. La pregunta que surge ahora es: ¿̨a qué refieren 'esto' y 'aquello'?

En Los problemas de la Filosofia (PF), texto de 1912, Russell (1973) afirma que "el ejemplo más evidente y notable del conocimiento por familiaridad" (p. 1088) son los datos de los sentidos; pero incluye, además, a las ideas abstractas o universales $^{2}$. La razón que da para ello es que, de lo contrario, nuestro conocimiento se restringiría solo a aquello que está presente a nuestros sentidos, por lo que no podríamos conocer cosas del pasado ni conocer verdades de ningún tipo. Ahora bien, respecto a la forma como los universales serían objeto de conocimiento por familiaridad, en $M L$ Russell (1973) dice lo siguiente: "No solo nos apercibimos de los amarillos particulares, sino que, si hemos visto un número suficiente de amarillos y tenemos la suficiente inteligencia, nos apercibimos del universal amarillo" (p. 1032).

Así, nos apercibimos de los universales en la medida en que, de alguna manera (si tenemos la suficiente inteligencia), somos capaces de pasar de los particulares que vemos a la idea abstracta o universal que, digámoslo así, recoge a todos esos particulares. Independientemente de lo problemática que pueda resultar esta propuesta (Giaquinto, 2012), nótese que la idea de que los universales son objetos de

2 Esta misma idea puede encontrarse en varios de sus escritos, por ejemplo, en su artículo de 1914, "Sobre la naturaleza del conocimiento directo" (Russell, 1981, p. 179) y en $M L$ (Russell, 1973, p. 1032). 
conocimiento por familiaridad depende, en últimas, de que damos por sentado que la apercepción de los universales se da a partir de la apercepción de los particulares y de que hemos aceptado que de estos últimos tenemos conocimiento por familiaridad. Pero, ¿qué lleva a Russell a decir que los datos de los sentidos son eso de lo cual tenemos conocimiento por familiaridad y, por tanto, son los referentes por excelencia de los términos 'esto' y aquello'? Como vimos, en $P F$ el autor toma por evidente este hecho (Russell, 1973, p. 1088). Sin embargo, más adelante, en $E l$ conocimiento humano, sus alcances y limites $(C H)$, texto de 1948 , va a dar un argumento a favor de esta idea. Dicho argumento afirma que de las sensaciones, como por ejemplo la cualidad 'rojo', tenemos conocimiento por familiaridad porque no tienen ningún carácter especialmente privado, es decir, porque son objetivas (Russell, 1983, p. 102), a diferencia de los casos de 'aquí' y 'ahora', los cuales son meramente subjetivos. Respecto de estos últimos Russell (1983) afirma:

Por otro lado, lo que yo llamo 'aquí es por necesidad diferente de lo que cualquier otro llama 'aquí, y lo que ahora yo llamo 'ahora' es por necesidad diferente de lo que llamo ahora' en otra ocasión y de lo que otro hombre llama 'ahora' en otros tiempos. Este es el punto destacado, en el lenguaje, del carácter esencialmente privado de la experiencia de cada individuo. (p. 102)

Para Russell, las sensaciones, a diferencia de las experiencia del aquí y del ahora, no son privadas, ya que "Se puede dudar si el género de sensación que yo llamo 'rojo' es similar al que otro hombre llama con igual nombre, pero no hay ningún fundamento positivo para suponer que haya una diferencia" (Russell, 1983, p. 102).

Aunque más adelante volveremos sobre este argumento, basta decir, por ahora, que lo afirmado hasta aquí muestra a Russell como un filósofo que sostiene una teoría de los términos como 'esto' y 'aquello' según la cual tales términos serían referenciales, en el sentido de que referirían directamente (sin la intermediación de una descripción) o bien a una entidad singular en el mundo, los datos de los sentidos, o bien a una entidad que está presente a la mente, como es el caso de los universales, que lo están gracias a su relación con los primeros. Si esto es así, esta teoría es una clara oposición a la postura que, al parecer, defiende Hegel en $F E$ y, en este sentido, podemos decir que el tema de los deícticos es uno más de los aspectos puntuales en los cuales Russell intentó tajantemente separarse de Hegel. En lo que sigue, haremos una reconstrucción de la manera como este 
último trata tales términos, haciendo énfasis en las diferencias con la postura de Russell, e intentando mostrar que en ningún caso él estaría respondiendo a los argumentos de Hegel, sino que se estaría quedando atrapado en lo que, hegelianamente hablando, podría llamarse el autoengaño de la certeza sensible.

En la introducción a la FE, Hegel describe su tarea en ese libro como el mostrar el camino de la conciencia desde la certeza sensible hasta el saber absoluto. Guiados por esta idea, y por algunas afirmaciones que hace el autor en la misma Introducción y al comienzo del capítulo sobre la certeza sensible (capítulo I), podemos decir que esa parte tiene como objetivo mostrar a la conciencia natural en su forma más simple e inmediata y, en este contexto, mostrar cómo la manera como ella misma se concibe es contraria a la manera como realmente opera cuando busca conocer, esto es, es contraria a su Verdad (Houlgate, 2013, pp. 32-33). Al respecto, la idea es que la conciencia, en la experiencia del día a día o en lo que podría considerarse como el primer estadio del conocimiento, se concibe a sí misma como captando al objeto directamente a través de los sentidos en el espacio y el tiempo; en este sentido, se concibe captando algo singular (einzeln) y no algo universal (allgemein). Así, la certeza sensible "piensa el objeto como un esto, aqui o un esto, ahora" (Houlgate, 2013, p. 32), y supone que ese esto, aqui y ese esto, ahora capturan un objeto único. En términos más ligados a la filosofía del lenguaje, podríamos decir que, en el nivel del lenguaje del día a día, las palabras 'esto,' 'aquí' y 'ahora' y, como veremos, también 'Yo' y el apuntar, se conciben como teniendo un referente específico, al que tenemos acceso directo a través de los sentidos, sin la mediación de un concepto o una descripción. A esto lo hemos llamado, usando los términos de Hegel, un singular.

Como puede verse, esta manera de entender el esto, el aquí, el ahora, el yo y el apuntar, es similar a la comprensión que tiene Russell de los términos 'esto' y 'aquello', en lo relativo a la referencia a entidades singulares (que él llama particulares). En este sentido, podemos decir que la teoría de Russell coincide con la manera como, según Hegel, la certeza-sensible se ve a sí misma. La cuestión es que, según el autor alemán, esta es solo la manera como ella se ve en un primer momento, pero no es su Verdad. Su verdad es que "aquello de lo cual la certeza 
sensible es explícitamente consciente" prueba ser "completamente universal" (Houlgate, 2013, p. 34). En resumen, el problema que Hegel va a plantear, para ponerlo en el contexto de la filosofía del lenguaje, es que los términos 'esto,' 'aquí,' 'ahora' y 'Yo', aunque se pretenden usar como términos singulares, en realidad resultan ser términos universales, ya que refieren no a un solo individuo (como lo haría el término singular), sino a una multiplicidad (como lo haría el término universal). Visto así, podría podría surgir la pregunta: ¿cuál es el meollo del problema si, en últimas, Russell también considera que cuando decimos 'esto' o 'aquello' podemos referirnos a universales?

Sobre tal interrogante, la tesis de Hegel solo es comprensible y diferenciable de la de Russell si se hace una distinción entre la manera como cada uno de ellos entiende los universales. Como vimos, Russell entiende un universal como una reunión de particulares (o singulares en la terminología de Hegel), en este sentido, la existencia de los universales depende de la existencia de esos particulares y de que podamos apercibirnos de ellos. Para Hegel, en cambio, parece ser que los universales no presuponen la existencia de los singulares, sino que aquellos existen por sí mismos, de tal manera que se instancian en la realidad. Entonces, donde Russell diría algo como 'esto es blanco', expresando una relación entre un particular y un predicado en la que el particular cae o no cae bajo el predicado, es decir, hace parte o no hace parte de la clase de las cosas blancas, Hegel diría algo como 'la blancura existe aquí' refiriéndose a que lo que tenemos al frente no es un individuo, sino una instancia, un ejemplo, de la blancura, del universal, que es, como veremos más adelante, una unidad indiferente a la multiplicidad de sus instanciaciones ${ }^{3}$.

Aclarado esto, podemos pasar a ver los argumentos que da Hegel a favor de su tesis. El autor presenta tres momentos de la experiencia de la conciencia, los cuales se pueden entender como tres argumentos que van en contra de la idea de que a través de los términos en cuestión la conciencia refiere a singulares, y, con ello, en contra de la idea de que la conciencia puede tener un conocimiento inmediato del mundo. En un primer momento, que corresponde a lo que Houlgate llama la primera experiencia de la certeza sensible, Hegel muestra que

3 Esta distinción la presenta Russell en su artículo de 1911 "Sobre las relaciones entre universales y particulares" (1981, p. 155-156), en el cual atribuye la primera teoría a aquellos que admiten tanto particulares como universales, y la segunda teoría a los que solo admiten la existencia de universales. 
tanto el esto como el ahora y el aquí son en realidad términos universales y no, como lo pretendía la conciencia, términos singulares. La certeza sensible comienza intentando responder a la pregunta iqué es el esto? Sin embargo, la pregunta ¿qué es el esto? se convierte en las preguntas iqué es el ahora? y iqué es el aquí?, pues, dice Hegel, los tres elementos (el esto, el aquí y el ahora) se encuentran interrelacionados. Para resumir el problema, cuando se intenta responder a la pregunta ¿qué es el ahora? cualquier cosa que digamos al respecto resulta ser falsa, si esa misma respuesta se da en otro momento. Lo cual muestra que el ahora es contextual, es decir, que lo que sea ese ahora depende de las circunstancias en que se enuncie (Hegel, 2010, p. 64). Esta contextualidad le indica a Hegel que el ahora es un universal y no un singular, pues está mediado por lo que el autor llama 'negatividades'. Al parecer, lo que se entiende por negatividades, en el caso del ahora, son los múltiples y diversos ejemplos que se pueden dar de lo que no es el ahora. Razón por la cual se dice que el ahora es universal. Sin embargo, lo que hace que el ahora sea un universal, en el sentido hegeliano, no es la mera multiplicidad de lo que no es el ahora, sino la manera como esa multiplicidad se relaciona con ese ahora, es decir, con la unidad. Para Hegel, lo que caracteriza dicha relación es el hecho de que la unidad es indiferente a esa multiplicidad. En sus palabras, "A una cosa así de simple, que es por la negación, que no es ni esto ni aquello, que es un no esto, e igualmente indiferente a ser aquello o esto lo denominamos un universal" (Hegel, 2010, p. 65).

Con base en lo anterior, podemos afirmar que el ahora es indiferente frente a las múltiples formas que pueda tomar aquello que el ahora no es. Esto, parece, quiere decir que el ahora, como tal, en realidad no refiere en particular a ninguna de esas formas que pueda tomar. En este sentido, el ahora no es una reunión de singulares que existen independientemente, sino una unidad que se puede instanciar, ejemplificar de diferentes maneras, pero cuya existencia no depende de las maneras en que pueda ser ejemplificada. Por tal razón, el ahora existe en cada uno de los momentos en que se puede instanciar, pero que existe independientemente de esas instanciaciones. Siguiendo a Dulckeit (2006, p. 158), podemos resumir el asunto de la universalidad del ahora sosteniendo que para que la conciencia pueda referir a un objeto por medio de un término como 'ahora' (ella en su texto en realidad habla del término 'esto'), ese objeto debe estar individuado, y para que pueda ser individuado, este debe estar determinado. La determinación involucra 
negación, puesto que para decir lo que algo es hay que distinguirlo de lo que no es. Al involucrar la negación, también se involucra una multiplicidad. Multiplicidad frente a la cual, como vimos, el término 'ahora' se muestra indiferente. En este sentido, para poder referir a un objeto con el término 'ahora', se necesita la intermediación de un universal entendido a la manera hegeliana.

Todo lo anterior lleva a Hegel a afirmar que los singulares supuestos por la certeza sensible inicial, es decir, los objetos que se dan a los sentidos, son inefables (unaussprechlich), puesto que no pueden ser dichos en el lenguaje, y esto se debe a que el lenguaje expresa lo que es verdadero y la verdad de la certeza sensible, por lo menos en lo que se ha visto en relación con el ahora, es su universalidad. Verdad, aquí, se contrapone a mera certeza, pues la conciencia está cierta de habérselas con singulares, pero en realidad tales singulares solo pueden ser expresados mediante universales. Y esto se debe a que tales singulares son ellos mismos universales. Visto así, podemos decir, entonces, que aquello a lo que Russell pretendería tener acceso, a saber, a los datos de los sentidos, que serían, por excelencia, los referentes singulares de los términos 'esto' y 'aquello', para Hegel resulta inefable, pues su experiencia está mediada por el lenguaje que es, en esencia, universal. Lo mismo ocurre con el aquí. Cuando decimos que el aquí es, por ejemplo, un árbol, y nos damos la vuelta, ese aquí ya no es un árbol sino una casa. De esta manera, el significado del aquí estaría dado por todas sus negatividades, esto es, por todas las circunstancias posibles de uso, que son negaciones unas de otras, opuestas entre sí, y frente a las cuales el aquí es indiferente.

En la segunda experiencia, la certeza sensible parte de suponer que el Yo puede funcionar como una especie de estabilizador, es decir, como algo que va a permitir que la referencia del aquí y del ahora se determinen, y que cada uno de tales términos refiera a un mismo singular. No obstante, acto seguido va a mostrar que el Yo, como en los demás casos considerados, no es un singular sino un universal. La idea, en líneas generales, es que la palabra 'Yo' puede corresponder a múltiples personas, dependiendo de las situaciones de uso, o, si lo dice una misma persona, puede corresponder a diferentes momentos de esa persona. En este sentido, el Yo funciona como un universal, al igual que el aquí y el ahora, ya que refiere a una 
multiplicidad, a todos los Yos, multiplicidad frente a la cual el término 'Yo' es indiferente y, en ese sentido, no permite estabilizar nada (Hegel, 2010, p. 66)4.

Otro argumento en contra de la singularidad de los deícticos es el que Hegel presenta en la tercera experiencia, la del apuntar. La certeza sensible parte del supuesto de que si apuntamos hacia algo cuando decimos 'esto,' 'ahora,' 'aquí' o 'yo,' el gesto de apuntar nos permitirá estabilizar la referencia de cada uno de esos términos. El problema es que, como lo anota Hegel, en el momento en que pretendemos señalar un ahora, un aquí o un Yo, lo que intentábamos señalar ya se ha ido, y a lo que estamos señalando es otra cosa. De esta manera, el apuntar, o lo que podríamos llamar definición ostensiva, tampoco permite responderle al universalismo que Hegel está planteando, o que se le presenta a la certeza sensible como su Verdad, pues cada vez que apunta señala a una multiplicidad y no a un singular. Todos los intentos fallidos de singularizar el 'esto', ya sea a través de su relación con el ahora y el aquí, o de su relación con el yo, o con el apuntar, llevan a la certeza sensible a concluir que el término 'esto' es un universal, "lo contrario de lo que aquella afirmación, [la original de la certeza sensible], asevera que es la experiencia universal" (Hegel, 2010, p. 69).

EN LA SECCIÓN ANTERIOR mostramos cuál es la teoría de los deícticos que se le podría atribuir a Hegel. En esa línea, lo que podemos decir es que, para el autor, tanto el término 'esto' como los otros deícticos que hemos considerado funcionan como términos universales y no como singulares. Considerarlos

4 Este elemento de universalidad parecería remitir a lo que David Kaplan, en su artículo "Demonstratives”, denomina 'carácter' (Kaplan, 1989, p. 505-507). Lo cual es un tipo de significado presente en los deícticos y otras expresiones, que el autor representa por medio de funciones que van de contextos posibles a contenidos, algo así como una regla que permite fijar el contenido de las expresiones en cada contexto en que son usadas (Braun, 1995). Esta es una sugerencia que nace de las anotaciones de uno de los pares evaluadores de este artículo, quien considera, además, la posibilidad de que dicha noción permitiera hacer compatibles la universalidad planteada por Hegel, la particularidad planteada por Russell y la mayoría de las teorías contemporáneas en torno a los deícticos. Este es un asunto que vale la pena explorar, pero que por ahora está por fuera del desarrollo de este artículo, del mismo modo que lo está la exploración de toda la presunta fuerza que tendrían las ideas de Hegel contrarias a Russell, y las discusiones que podrían suscitarse entre aquel y los lingüistas contemporáneos. 
singulares, o como universales de los cuales nos apercibimos en la medida en que nos apercibimos de los singulares, como curiosamente más tarde lo va a hacer Russell con el término 'esto', es simplemente una manera de quedarse atrapado en el autoengaño de la certeza sensible. Y esto es 'curioso' porque, tal como se señaló al comienzo de este artículo, Russell fue hegeliano durante un buen período de su historia intelectual; y si lo que se ha mostrado en este trabajo es correcto, entonces, cabe preguntarse por la manera en que él estaba entendiendo la $F E$ y, en particular, el capítulo de la certeza sensible pues, pese a todo, termina cayendo en el problema al cual el mismo Hegel apunta.

Finalmente, volviendo al asunto de los deícticos, es relevante considerar que tal vez el problema de fondo entre los dos autores sea que, mientras Russell va a mantener lo que en el contexto de la filosofía de proceso (Process Philosophy) se conoce como una metafísica substancialista, Hegel va a sostener una metafísica de procesos o, en otros términos, una ontología, en la medida en que, para Hegel, los principios de la realidad no se distinguen de los principios racionales. Una diferencia fundamental entre ambas posturas es que los primeros, los substancialistas, considerarían que la realidad está formada por objetos estables; mientras que los procesualistas dirían que nada de lo que existe en el mundo, incluso las ideas, es un objeto estable "sino un elemento procesual que está en tránsito y que no puede ser propiamente entendido a través de sus propiedades estables o de una sucesión de estados estables, un asunto de ahora esto, ahora lo otro" (Rescher, 1996, p. 13). Si esto es así, la discusión acerca de los deícticos estaría marcada por el hecho de que, en ella, los dialogantes adoptan puntos de partida que son maneras de ver la realidad completamente diferentes. Así, mientras Russell ve una realidad estable, en la que los datos se diferencian claramente unos de otros, Hegel ve procesos que implican el cambio constante, la contradicción y la existencia de un continuo. Algo de lo anterior puede encontrarse en el hecho de que, mientras Russell ve el término 'esto' como algo estable porque sus referentes, las sensaciones, a diferencia de los referentes de los términos 'aquí y 'ahora', son algo objetivo, Hegel lo ve desde el inicio como algo que se mueve, cuando dice que la pregunta ¿qué es esto? debe responderse respondiendo a las preguntas ¿qué es el aqui? y ¿qué es el ahora?, según las cuales el aquí y el ahora se mueven permanentemente.

Con todo, esta discusión sobre los deícticos trata del establecimiento de posiciones básicas fundantes encontradas, en las que ninguno de los dos autores da 
argumentos que permitan justificar su posición. Por un lado, Hegel presenta como algo evidente la idea de que la realidad a la cual se refieren los términos 'esto', 'aquí, 'ahora' y 'yo', y que se pretende capturar con el acto de mostrar, se mueve permanentemente. En este sentido, no da razones que sustenten la idea de que la realidad es algo cambiante, es decir, que lo que las cosas son en un segundo no lo son al segundo siguiente, y que estos cambios ocurren en una especie de continuo, idea que, al parecer, es la que se encuentra a la base de sus argumentos relativos a la universalidad de los deícticos. Por otro lado, aunque Russell intenta justificar su afirmación de que las sensaciones son algo objetivo (y por lo tanto estable), lo que le permite afirmar que el término 'esto' tiene un referente singular, lo hace con un argumento que, a nuestro modo de ver, resulta débil. En efecto, como se recordará, el argumento de Russell es el siguiente: "se puede dudar si el género de sensación que yo llamo "rojo" es similar al que otro hombre llama con igual nombre, pero no hay ningún fundamento positivo para suponer que haya una diferencia" (Russell, 1983, p. 102). Y este argumento es débil porque parece tener la forma de un argumento por ignorancia: en él se asume una cierta tesis, a saber, que no hay ninguna diferencia entre el género de sensación que yo llamo 'rojo' y el que otra persona llama con igual nombre, dado que no se ha podido probar la tesis contraria, esto es, dado que no se ha podido comprobar positivamente que exista de hecho alguna diferencia. El problema es que lo mismo podría decir quien intentara sostener la tesis contraria. Esa persona sostendría, quizá, que el género de sensación que yo llamo 'rojo' es diferente de lo que otra persona llama con el mismo nombre, acudiendo para ello al hecho de que lo contrario, que sea lo mismo, no ha sido probado.

Lo anterior indica que la debilidad de este argumento no es que Russell no sepa argumentar, ni más faltaba, sino que, para él, no es muy importante argumentar a favor de su tesis de la estabilidad de las sensaciones, pues, que la realidad esté formada por cosas estables y distinguibles unas de otras es algo, digamos, evidente, así como para Hegel es evidente que la realidad es toda, incluso en lo relativo a las sensaciones, una realidad cambiante. Algo parecido puede decirse de sus posiciones en relación con los universales. Al respecto, Hegel ve una realidad, el universal, que se manifiesta de diferentes maneras en diferentes momentos y lugares; por su parte, Russell ve diferentes realidades, los particulares, que se reúnen bajo un universal. Y sobre ello ninguno de los dos da argumentos que sustenten 
sus afirmaciones. Más aún, parecería que en el uso que les dan, cayeran en una especie de circularidad. En el caso de Hegel, por ejemplo, puede verse que su argumentación a favor de la universalidad de los deícticos depende de suponer que los universales son universales en el sentido hegeliano del término, es decir que son realidades que se instancian y no reuniones de singulares, pues los singulares no existen. La prueba de que los singulares no existen es que los mismos deícticos manifiestan ser universales en el sentido hegeliano. En el caso de Russell, uno podría decir que si él quisiera responder a Hegel argumentando en contra de la existencia de particulares (singulares), tendría que acudir a su propia noción de universal para mostrarle que aunque es cierto que la experiencia de particulares resulta ser experiencia de universales, esos universales son, ni más ni menos, reuniones de particulares. Sin embargo, aunque dicha circularidad no sea un problema de argumentación, muestra que cada uno de los autores asume una cierta visión del mundo como evidente y no encuentra necesario argumentar a favor de ella.

Basándonos en lo anterior, afirmamos que tal vez el problema no esté en la manera como cada uno de estos filósofos entiende los llamados deícticos, sino en su trasfondo ontológico. Es el terreno ontológico sobre el cual están montadas sus teorías acerca de los deícticos lo que hace que tales teorías sean tan disímiles. En este sentido, volviendo a lo que hemos llamado el autoengaño de la certeza sensible, podemos decir que este no refiere solo al autoengaño en relación con el esto, el aqui, el ahora, el yo y el mostrar, sino, desde la perspectiva de Hegel, al autoengaño de creer que la realidad pueda ser dividida en partes y que estas sean estables, cuando lo que la experiencia nos muestra es que esa realidad es un permanente fluir, un cambio continuo, un proceso que no se acaba. La cuestión de que cómo sea esa realidad 'realmente' es algo que no ha sido demostrado, y que tal vez no pueda serlo, mucho menos a través de la experiencia, sino que es lo que constituye el supuesto sobre el cual está montado todo el resto del aparataje teórico, inclusive aquello que consideramos como nuestra experiencia. $Y$ esto vale tanto para la postura de Hegel, para quien la realidad es algo cambiante, como para la de Russell, para quien la realidad es algo estable 5 .

5 Uno de los pares evaluadores de este artículo manifestó que en este punto habría un problema al apelar a una diferencia ideológica profunda entre la ontología substancialista de Russell y el procesualismo de Hegel para explicar las diferencias en la manera como cada uno de ellos ve el problema de los deícticos. La dificultad consistiría en que, según el par académico, "un indéxico como 'esto' 
Lo anterior significa que no solo a Hegel se aplicaría la frase de que para aceptar lo que dice hay que aceptar de entrada sus presupuestos ontológicos, en este caso, su procesualismo ${ }^{6}$, sino que también, para aceptar lo que Russell dice, habría que aceptar su substancialismo. Esto, obviamente, va en contravía de una afirmación bastante certera que hace Russell en su artículo de 1924, titulado "Atomismo lógico" (Russell, 1981, p. 455), según la cual, lo que es fundamental en su filosofía no es el realismo, como algunos lo han querido sugerir, sino su posición respecto a la lógica, ya que es sobre la lógica que está montado su realismo y no al revés, lo cual tiene como consecuencia que él podría cambiar su posición en lo que se refiere a la disputa entre realistas y sus adversarios, pero no su posición frente a la lógica que es, en ese momento y hasta el final, una lógica atomista. Lo único que podríamos sostener al respecto es que habría que revisar si es cierto que su lógica atomista se sostiene sin el supuesto de que en la realidad hay porciones estables como, por ejemplo, las sensaciones, o cualesquiera otras. Ya está visto que en lo que se refiere a los deícticos parece no ser este el caso, pero en lo que se refiere a las proposiciones atómicas habría que averiguarlo.

\section{Referencias}

Braun, D. (2012). Indexicals. E.N. Zalta (Ed.), The Stanford Encyclopedia of Philosophy (Summer Edition). Versión Online: http://plato.stanford.edu/archives/sum2012/entries/indexicals/ Recuperado el 30.03.14.

Braun, D. (1995). What is Character? Journal of Philosophical Logic, 24, pp. 227-240.

no designa menos un particular cuando se usa para referir a un proceso o a una eventualidad (una batalla o un partido de fútbol) que cuando se trata de referir a una maceta". La formulación de este problema presenta el obstáculo de que al parecer se está entendiendo un proceso en términos de porciones estables de la realidad que podemos determinar en dónde comienzan y en dónde terminan, cuando la manera como se entendería un proceso desde el punto de vista del procesualismo no permitiría establecer estos puntos de referencia, pues la realidad para ellos sería un continuo fluir. Es decir que el 'esto', referido a una realidad permanentemente cambiante, no podría referir ni a partidos de fútbol o batallas ni tampoco a macetas, puesto que no habría en ningún caso una realidad estable a la cual hacer referencia. Con entero agradecimiento esperamos haber respondido a la objeción planteada por el evaluador del artículo.

6 Otros hablarían más bien de su idealismo, pero yo considero más básico en Hegel el procesualismo que el idealismo, en la medida en que si el idealismo fuera lo más básico, él muy bien podría ser un idealista para el que las ideas son algo estático, lo cual no parece ser el caso. 
Dulckeit, K. (2006). Language, Objects, and the Missing Link. Toward a Hegelian Theory of Reference. J. O'Neill Surber (Ed.), Hegel and Language (pp. 145-164). New York: SUNY Press.

Giaquinto, M. (2012). Russell on Knowledge of Universals by Acquaintance. Philosophy, 87(4), pp. 497-508.

Hegel, G.W.F. (2010). La Fenomenología del Espiritu. Madrid: Abada EditoresUAM Ediciones.

Houlgate, S. (2013). Hegel's Phenomenology of Spirit. London: Bloomsbury.

Hylton, P. (1990). Russell, Idealism and the Emergence of Analytic Philosophy. Oxford: Clarendon Press.

Kaplan, D. (1989). Demonstratives. J. Almog, J. Perry \& H. Wettstein (Eds.), Themes from Kaplan (pp. 481-564). New York/Oxford: Oxford University Press.

Klement, K. (2014). Russell's Logical Atomism. E.N. Zalta (Ed.), The Stanford Encyclopedia of Philosophy. Versión Online:http://plato.stanford.edu/archives/spr2014/entries/logical-atomism/ Recuperado el 30.03.14.

Rescher, N. (1996). Process Metaphysics, An Introduction to Process Philosophy. New York: SUNY Press.

Russell, B. (1983). El conocimiento humano (Trad. N. Míguez). Madrid: Ediciones Orbis S. A.

Russell, B. (1981). Sobre la naturaleza del conocimiento directo. Lógica y Conocimiento (pp. 175-244). (Trad. J. Muguerza). Madrid: Taurus.

Russell, B. (1981). La filosofía del atomismo lógico. Lógica y Conocimiento (pp. 245-396). (Trad. J. Muguerza). Madrid: Taurus.

Russell, B. (1981). Atomismo Lógico. Lógica y Conocimiento (pp. 451-484). (Trad. J. Muguerza). Madrid: Taurus.

Russell, B. (1981). Sobre las relaciones entre universales y particulares. Lógica y Conocimiento (pp. 145-174). (Trad. J. Muguerza). Madrid: Taurus.

Russell, B. (1973). Los problemas de la filosofía. Bertrand Russell, obras completas, Tomo II (pp. 1067-1143). (Trad. J. García-Puente). Madrid: Editorial Aguilar. Russell, B. (1973). Misticismo y lógica. Bertrand Russell, obras completas, Tomo II (pp. 919-1030). (Trad. J. García-Puente). Madrid: Editorial Aguilar.

Stern, R. (2002). Hegel and the Phenomenology of Spirit. London: Routledge. 